\title{
Unidirectional mechanism for reentrant activity generation in excitable media
}

\author{
Irene Sendiña-Nadal, ${ }^{1,2 *}$ Maite de Castro, ${ }^{3}$ Francesc Sagués, ${ }^{4}$ and Moncho Gómez-Gesteira ${ }^{3}$ \\ ${ }^{1}$ ESCET, Universidad Rey Juan Carlos, Tulipán, s/n. 28933 Móstoles, Spain \\ ${ }^{2}$ Grupo de Física non Lineal, Facultade de Física, Universidade de Santiago de Compostela, 15706 Santiago de Compostela, Spain \\ ${ }^{3}$ Facultade de Ciencias, Campus de Ourense, Universidade de Vigo, 32004 Ourense, Spain \\ ${ }^{4}$ Departament de Química Física, Universitat de Barcelona, Diagonal 647, 08028 Barcelona, Spain
}

(Received 3 October 2001; revised manuscript received 1 May 2002; published 25 July 2002)

\begin{abstract}
A closed excitable pathway with one point-to-point connection is used to generate a rotating wave both in experiments using the photosensitive Belousov-Zhabotinsky system and numerically with an Oregonator reaction-diffusion model. By varying the excitability and geometrical properties of the medium, propagation can be made unidirectional or bidirectional, giving rise, respectively, to the existence or not of sustained reentrant activity in a closed excitable track.
\end{abstract}

DOI: 10.1103/PhysRevE.66.016215

PACS number(s): 05.45.-a, 82.40.Ck, 47.20.Ky

Sustained spatiotemporal activity is found in different types of autocatalytic chemical reaction and in biological systems [1]. In many cases it can be attributed to a source of recurrent excitation, such as a pacemaker node that generates a healthy heartbeat [2]. In the absence of the localized source, an ongoing activity itself can sometimes provide the necessary recurrent excitation, either by recirculation of the excitation through a reentrant path or through a topological defect in the wave front, such as the tip of a spiral wave. Both of these mechanisms have been implicated in the disturbances of the cardiac rhythm. Spatial differences in the recovery properties, invasion of a wave into a vulnerable region of the preceding wave [3-5], as well as sharp boundaries or obstacles [6-9] are all known to generate spiral waves. Slow conduction and the existence of a unidirectional transiently blocked region are other mechanisms responsible for the genesis of reentrant wave fronts and spiral waves [10-12].

These types of reentrant activity and rotating wave structures can also be readily observed in chemical systems: pinwheels [13] or excyclons [14] in an annular reactor, and chemical clocks in triangular or square shaped domains [15]. However, the reentrant activity in such experiments is usually created in a manner that is somewhat artificial. Since any perturbation results in two fronts propagating in opposite directions, these fronts, if left to themselves, annihilate each other half the circulation period later. In order to produce a sustained activity, one of them has to be artificially eliminated to allow the other one to circulate unhindered.

Here we explore how an asymmetry in the system can produce a self-sustained reentrant wave from a single initial perturbation. We consider a closed excitable pathway with a constriction. If the initial perturbation of precisely defined small amplitude is injected on one side of the constriction, it will be able to propagate only along the open track but not through the constriction, thus creating a single reentrant wave in the closed pathway. We will present experimental evidence of such unidirectional injection further corrobo-

\footnotetext{
*Corresponding author. Email address: isendina@venus.escet.urjc.es
}

rated by numerical simulations, together with an analysis with additional numerical studies of detailed aspects of the unidirectional behavior.

We use a spatially extended Belousov-Zhabotinsky (BZ) medium catalyzed by the ruthenium (II)-bipyridyl
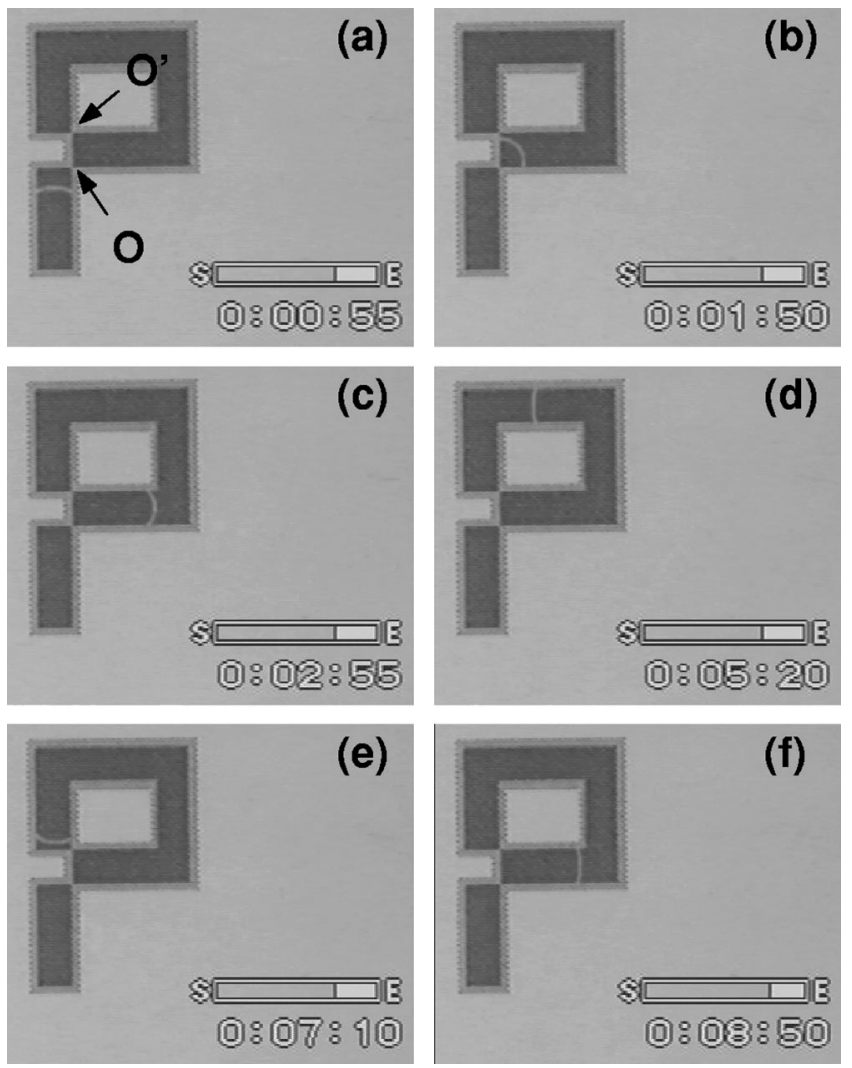

FIG. 1. Sequence of images showing the time evolution of a counterclockwise rotating wave in the BZ reaction starting from (a). Panel (a) shows the special points marked as $O$ and $O^{\prime}$. The dimensions of the closed path are $0.9 \mathrm{~cm} \times 0.9 \mathrm{~cm}, R=2 \mathrm{~mm}$, and the light intensity inside it is $107.8 \mu \mathrm{W} / \mathrm{cm}^{2}$. Gel: solution of $15 \%$ sodium silicate, $0.71 \mathrm{mM} \mathrm{Ru}(\mathrm{bpy})_{3}^{2+}$, and $0.18 \mathrm{M} \mathrm{H}_{2} \mathrm{SO}_{4}$; preparation as in [18]. Catalyst-free $\mathrm{BZ}$ solution initial concentrations: $0.18 \mathrm{M} \mathrm{KBr}, 0.33 \mathrm{M}$ malonic acid, 0.39 $\mathrm{M} \mathrm{NaBrO}_{3}$, and $0.69 \mathrm{M}$ $\mathrm{H}_{2} \mathrm{SO}_{4}$. 


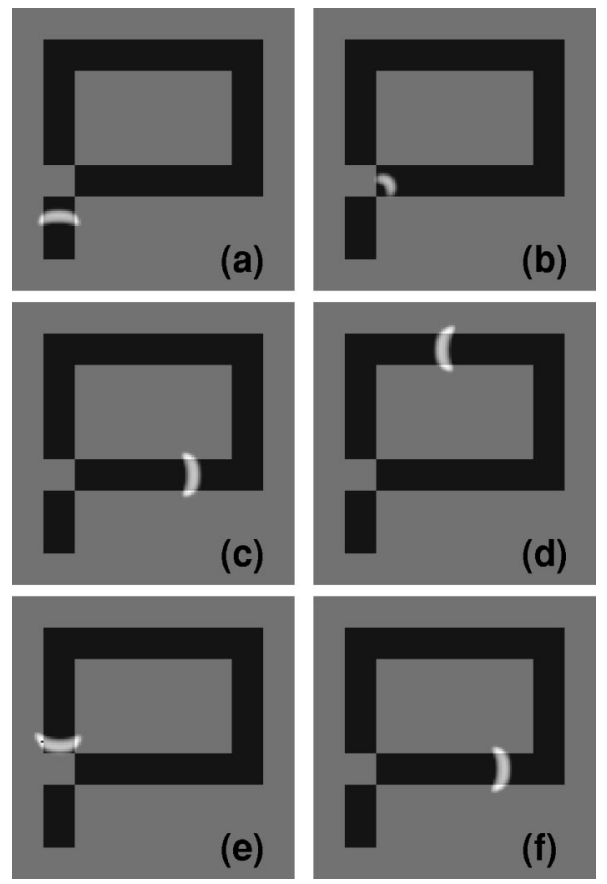

FIG. 2. Sequence of images showing the time evolution of a counterclockwise rotating wave from a numerical simulation of the model given by Eq. (1). Times: (a) 0.3, (b) 0.9, (c) 2.1, (d) 4.8, (e) 6.6 , and (f) 8.25 t.u. The closed path has $105 \times 75$ grid points, and $R=15$ grid points. Parameters of the model are $\varepsilon=0.01, f=3$, and the values of the parameter $\phi$ inside and outside the labyrinth are 0.02 and 0.0318 , respectively.

$\left[\mathrm{Ru}(\mathrm{bpy})_{3}^{+2}\right]$ complex, which is sensitive to visible light [16] thus allowing spatial control of the excitability of the system [17]. The Ru complex is immobilized in a silica gel matrix $1 \mathrm{~mm}$ thick and bathed in the BZ solution with concentrations given in Fig. 1. The temperature is kept constant at $25 \pm 1{ }^{\circ} \mathrm{C}$. White light (190 W halogen lamp) passes first through a diffusion screen, then reaches the gel, the interference filter $(450.6 \mathrm{~nm}$; transmission $56 \%$ ), and, finally, the video equipment for image recording. The video images are processed through an image-acquisition card and analyzed on a personal computer.

An excitable path is produced by projecting onto the gel a computer generated image shown in Fig. 1. There, lower illumination creates excitable regions surrounded by unexcitable medium. An initial perturbation is generated and allowed to propagate upward through a straight corridor as shown in Fig. 1(a). The corridor is joined at a corner point $O$ to a rectangular path such that the incoming wave propagates into this path as shown in Fig. 1(b). The key event occurs during the transition from (b) to (c), where the wave front propagates to the right but not through the second corner contact $O^{\prime}$ in the immediate vicinity of the first one. This mechanism is responsible for unidirectional injection of excitation into the ring path. At every cycle the wave passes through $O^{\prime}$ as shown in (e) and (f) and sustains the activity in the ring. As seen here, the wave does not pass back through $O$, and thus is not reemitted back to the corridor where the initial perturbation was generated.
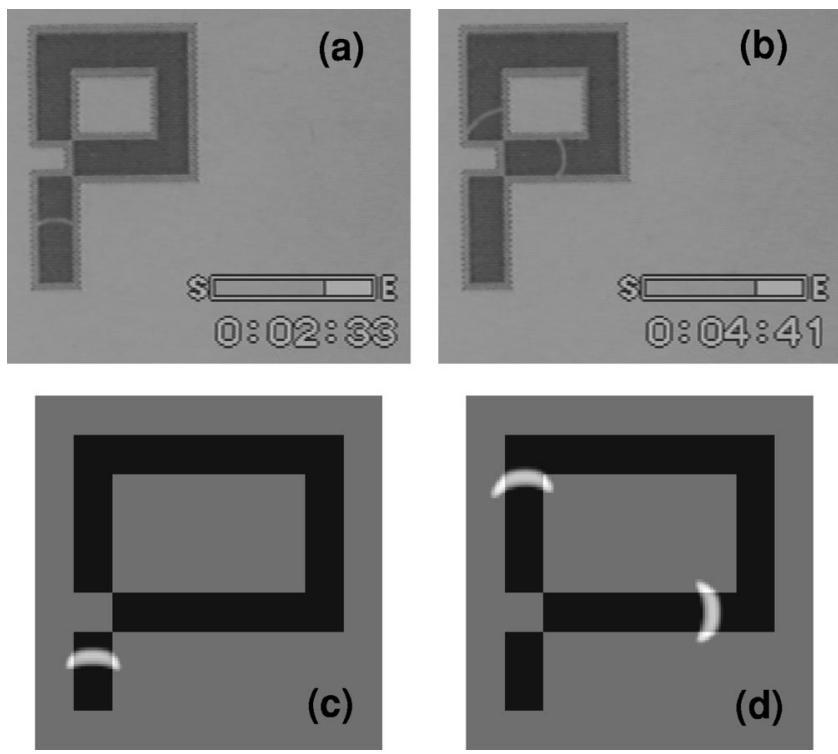

FIG. 3. (a), (b) Bidirectional propagation in a closed path in the excitable BZ reaction. Light intensity inside the labyrinth $100.25 \mu \mathrm{W} / \mathrm{cm}^{2}$. Other parameters as in Fig. 1. (c),(d) Bidirectional propagation in a closed path simulated with the model given by Eq. (1). $\phi_{\text {in }}=0.02, \phi_{\text {out }}=0.0308$. Other parameters as in Fig. 2.

Numerical simulations of the previous experimental situation are performed with the two-variable Oregonator model $[19,20]$, modified to include the photosensitivity of the BZ reaction [21]:

$$
\begin{gathered}
\frac{\partial u}{\partial t}=\frac{1}{\varepsilon}\left(u-u^{2}-(f v+\phi) \frac{u-q}{u+q}\right)+D_{u} \nabla^{2} u, \\
\frac{\partial v}{\partial t}=u-v,
\end{gathered}
$$

where the dimensionless variables $u$ and $v$ correspond to $\mathrm{HBrO}_{2}$ and the $\mathrm{Ru}(\mathrm{II})$ catalyst concentrations, respectively, and $D_{u}=1$ is the scaled diffusion coefficient. Since the $\mathrm{Ru}$ (II) is bound to the silica gel medium, there is no corresponding diffusion term for $v$. The parameter $\phi$ corresponds to the light-induced production of $\mathrm{Br}^{-}$, which is directly proportional to the intensity of the illumination such that the excitability and wave velocity decrease with increasing $\phi$. The system (1) is numerically integrated using an Euler method with a time step $\Delta t=10^{-3}$ t.u. (time units) and a grid size $\Delta s=0.16$ s.u. (space units) in an array of 135 $\times 135$ points and with zero flux boundary conditions. The Laplacian operator is solved by following a nine-point scheme [22].

Numerical results for the same mode of propagation as in Fig. 1 are shown in Fig. 2 where an initial perturbation [Fig. 2(a)] gives rise to a counterclockwise circulating wave [Figs. 2(b)-2(f)]. By increasing the excitability within the labyrinth, the propagation mode becomes bidirectional, where an initial wave front reaching the second contact vertex [point $O^{\prime}$ in Fig. 1(a)] is able to cross it. This time, clockwise and counterclockwise rotating waves will exist until they annihi- 


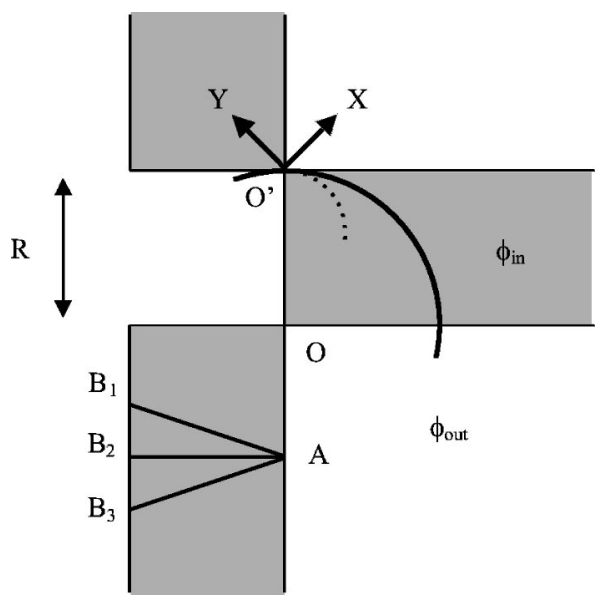

FIG. 4. Sketch of the part of the closed track operating like a diode. The dark area represents the track and the lighter one the outer medium.

late at some point on the track, Fig. 3, both experimentally [(a) and (b)] and numerically [(c) and (d)].

The behavior shown in Figs. 1 and 2 depends on the ability of the circulating wave to pass through a corner contact and its inability to pass through two such contacts successively if they are placed at a close distance from each other. Figure 4 shows the arrangement of the two contacts and the parameters that we shall consider in explaining this behavior. There is a clear difference between the waves approaching $O^{\prime}$ depending on their origin. In Figs. 1 and 2 the wave already circulating in the path exhibits much lower curvature than the one that has just exited from the corner contact $O$.

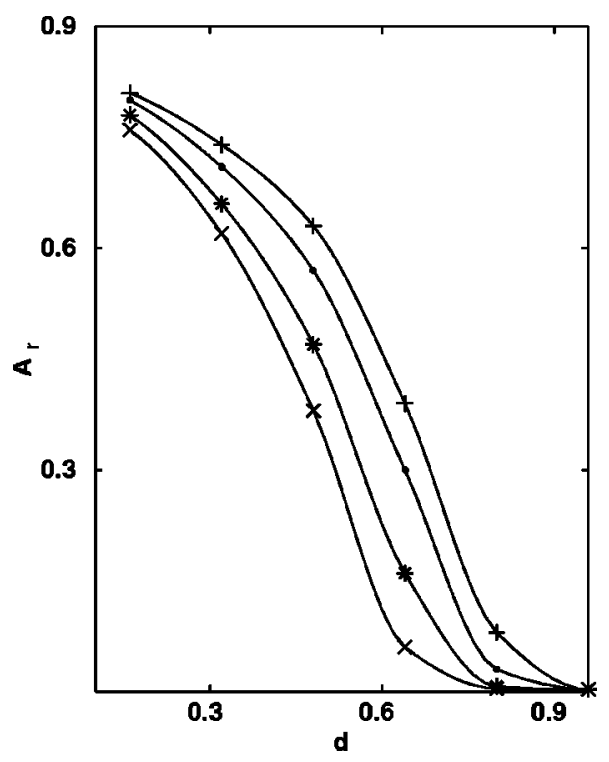

FIG. 5. Dependence of the relative amplitude on the distance from the track measured in space units. The relative amplitude $A_{r}$ $=A_{d} / A_{0}$, where $A_{0}$ is the maximum value of the activator at the center of the track and $A_{d}$ is the maximum at a distance $d$ from the track, is observed to decrease with increasing $d$ and $\phi_{\text {out }}$. Calculations were carried out for a constant $\phi_{\text {in }}=0.02$ and different $\phi_{\text {out }}$ values $[0.0306(+), 0.0310(\bigcirc), 0.0314(*)$, and $0.0318(\times)]$.

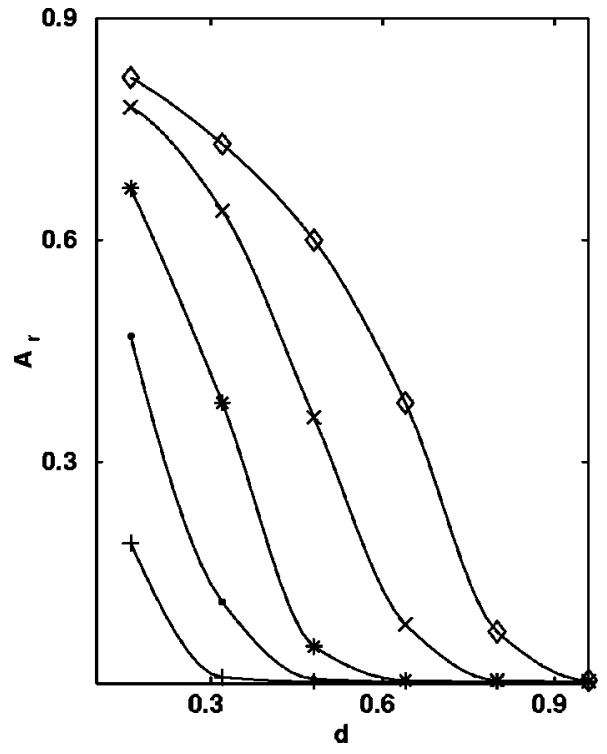

FIG. 6. Dependence of the relative amplitude on the distance $d$ from the track (in s.u.) for different radii $R[1.36$ s.u. $(+), 2.03$ s.u. $(\diamond), 2.70$ s.u. $(*), 4.75$ s.u. $(\times)$, and 9.05 s.u. $(\diamond)]$. At any $d$, the relative amplitude of the front is observed to increase with the distance from the emission point (corner $O$ ). The length of the front in the outer region corresponding to $A_{r}$ above some critical value considerably decreases as $R$ decreases. Calculations were carried out for constant $\phi_{\text {in }}=0.02$ and $\phi_{\text {out }}=0.0305$ values.

The ends of the former visibly "spill" out of the excitable track while the latter is contained entirely inside the channel. To quantify these factors and show that they indeed play a decisive role in shaping the observed behavior we have conducted a series of additional numerical experiments measuring the amount of activator species spreading outside the channel. The initiation of the wave requires the concentration of the activator species to exceed some threshold value which mainly depends on the excitability of the medium. This initiation is related to the amount of activator reaching across the corner contact by spreading outside the track from the propagating wave. Measurements of the spreading as a function of the distance from the track boundary are shown in Fig. 5. The relative amplitude outside the track $A_{r}(d)$ is defined as the ratio between the maximum value of the activator at a distance $d$ transversely from the track border and the maximum at the center of the track. To evaluate this quantity, a planar wave front (generated from $B_{2}$ to $A$ in Fig. 4) was allowed to propagate along the first part of the track from its bottom. The excitability inside the track was kept constant and $A_{r}(d)$ was measured for different values of $\phi_{\text {out }}$. After 1.9 t.u., and before the wave front reached the corner $O$, the relative amplitude was observed to decrease with increasing distances as shown in Fig. 5. In addition, this amplitude also decreases when increasing $\phi_{\text {out }}$, although the penetration length in the outer medium hardly varies with $\phi_{\text {out }}$. Penetration into the outer medium also depends on the distance from the emission point as is shown in Fig. 6. This plot was obtained by means of a planar wave front (generated from $B_{2}$ to $A$ in Fig. 4) which after crossing the corner $O$ becomes an arc. The relative amplitude, measured along 


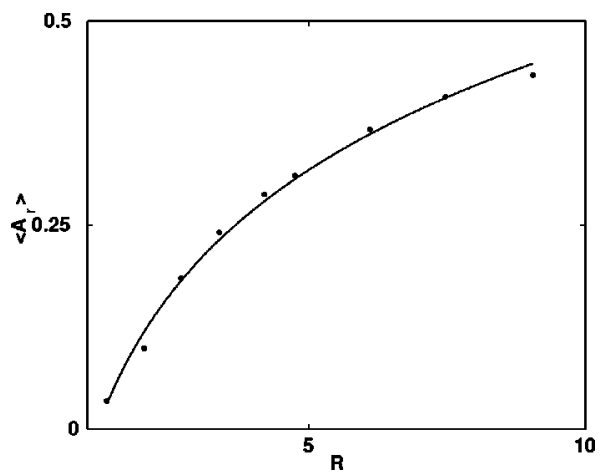

FIG. 7. Mean relative amplitude dependence on $R$. The relative amplitude of the front outside the track was averaged over a length equal to $0.96 \mathrm{~s}$.u. The mean relative amplitude is observed to increase with $R$ according to $\left\langle A_{r}\right\rangle=0.22 \ln (R)+0.036$. Calculations were carried out with constant $\phi_{\text {in }}=0.02$ and $\phi_{\text {out }}=0.0305$.

the wave front at the instant shown in Fig. 4, is observed to increase with $R$. The penetration length in the outer medium strongly depends on $R$, such that for large $R$ values the extent of the wave front outside the excitable track remains relatively constant while for small $R$ values it is greatly reduced. If the excitability inside and outside the propagation channel is appropriately chosen, planar waves will propagate successfully through a corner contact, while curved waves will cause only subthreshold excitation and will fail to propagate.

The contribution of the wave front to induce an excitation at the other side of the corner point $O^{\prime}$ depends not only on the extent of the wave outside the track but also on the amplitude of the wave front. By averaging over the length of the front beyond the border we obtain the mean activator amount $\left\langle A_{r}\right\rangle$ which is found to increase as $\ln (R)$, as shown in Fig. 7. There is also a secondary effect related to the symmetry of the front with respect to the obstacle. Intuitively it can be attributed to "the amount of front propagating in the passage direction." Comparing the arc profiles shown in Fig. 4, it is clear that the solid front, corresponding to a larger value of $R$, approaches the corner $O^{\prime}$ having more points with a nonvanishing positive component of the normal velocity along the axis $Y$, which favors crossing through $O^{\prime}$. To analyze this

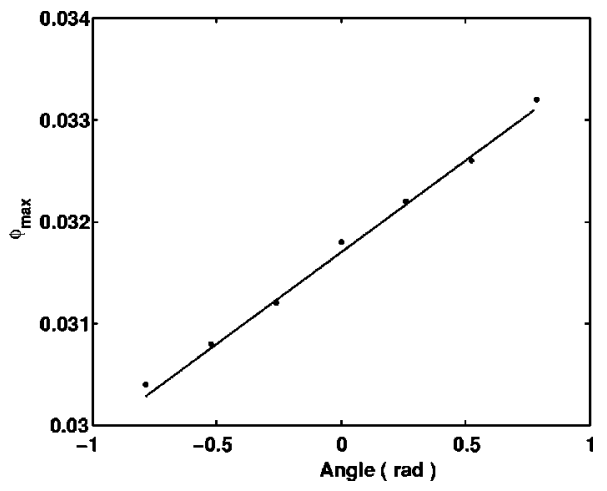

FIG. 8. Maximum $\phi_{\text {out }}$ allowing propagation through corner $O$ as a function of the initial angle. For a constant $\phi_{i n}=0.02$, the maximum $\phi_{\text {out }}$ is observed to increase when increasing the angle. Positive angles correspond to $Y\left(B_{i}\right)>Y(A)$ (see Fig. 4).

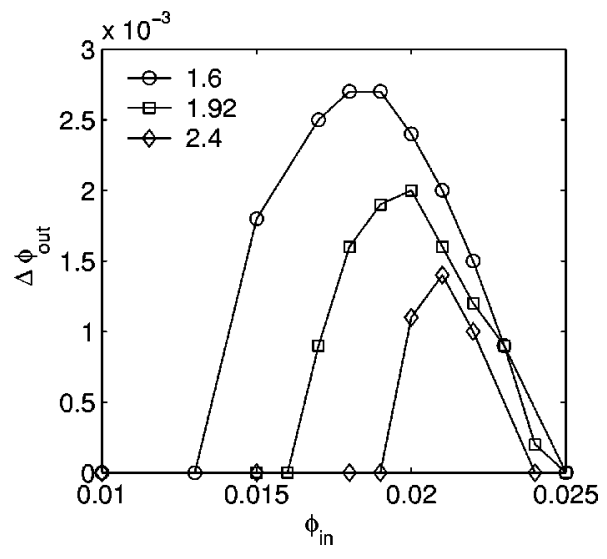

FIG. 9. Range of excitabilities outside the track, for which unidirectional propagation exists, as a function of the excitability inside the track, $\phi_{i n}$, for three different widths $R$ (in space units). The window size was calculated as the difference between the values of the parameter $\phi$ outside the track where the transitions from bi- to unidirectional propagation and from unidirectional to no propagation occur. Numerical parameters are the same as in Fig. 2.

effect we have conducted the following numerical experiment. A planar wave front was generated between $A$ and $B_{i}$ in Fig. 4 with the choice of $B_{i}$ determining the initial angle of wave propagation. The distance between point $A$ and the corner $O$ is kept constant. The excitability $\phi_{\text {in }}$ was fixed and $\phi_{\text {out }}$ increased in successive iterations until the front failed to propagate through the corner $O$. Figure 8 shows how the maximum $\phi_{\text {out }}$ allowing front propagation through the corner increases when increasing the initial angle, that is, when the propagation direction of the front approaches the passage direction through the corner.

To investigate the range of parameters resulting in unidirectional injection we have systematically varied $\mathrm{R}, \phi_{\text {in }}$, and $\phi_{\text {out }}$. For reasonably small distances between the corners $O$ and $O^{\prime}$ and fixed excitability $\phi_{\text {out }}$ outside the track, changing the value of $\phi_{\text {in }}$ results in the following succession of behaviors.

(1) For small values of $\phi_{\text {in }}$ the medium is so excitable that a minimal perturbation reaching the corner point $O^{\prime}$ can pass through. This behavior is shown in Fig. 3, where an initial wave front is able to cross the second corner point $O^{\prime}$. The resulting counterpropagating waves will later annihilate each other at some point on the track.

(2) For larger values of $\phi_{\text {in }}$ a wave front can propagate through the corner $O$ but not through the corner $O^{\prime}$, as was presented earlier.

(3) For even larger values of $\phi_{\text {in }}$ the medium becomes so little excitable that a wave front cannot cross over the first corner point $O$ and no interesting behavior arises in this case.

The interval of excitability $\Delta \phi_{\text {out }}$ outside the track that bounds the window of unidirectional injection for given values of excitability $\phi_{i n}$ inside the track and $R$ is shown in Fig. 9. The excitability window resulting in unidirectional injection becomes narrower with increasing $R$ and disappears completely above a certain critical value of $R$. For distances between the corners $O$ and $O^{\prime}$ above this critical value the increase in excitability changes the behavior from absence of 
any propagation through the corner to propagation through two successive corners without the intervening region of unidirectional injection.

Thus the unidirectional propagation is the result of both geometrical and chemical constraints. A threshold exists, depending on the medium excitability $\phi_{i n}$, for the amount of activator for the wave to penetrate through the corner point. This amount of activator which diffuses outside the track from a propagating wave depends upon the wave curvature as shown in Fig. 7, and to a lesser extent on the outside excitability $\phi_{\text {out }}$, Fig. 5. Low curvature waves circulating in the track easily pass through the constriction, but if a second constriction $O^{\prime}$ is placed close enough to the first $O$, the highly curved wave exiting from the first constriction $O$ does not propagate far enough outside the track to pass through the second constriction.

We have demonstrated how a rotating wave in a closed excitable pathway can be naturally initiated, and presented a detailed analysis of the unidirectional injection mechanism. We must note the similarity of some of the effects observed in our experiments to the findings from electrophysiological experiments in cardiac muscle [10] and arrays of electronic circuits [23] that were designed to study cardiac propagation at the microscopic level. Spach et al. in [10] demonstrated that a block in a region could be turned on or turned off simply by changing the direction of an excitation wave front with respect to the axis of the fibers. In particular, they concluded that the unidirectional block is not only due to differ- ences in membrane properties but also dependent upon the angle from which the excitation wave front enters the fibers, which is very similar to the behavior presented in Fig. 8.

Unidirectional propagation $[24,25]$ has recently been reported in relation to computing logic operations [26]. It was made possible by introducing a "diode" [27] based on the asymmetrical arrangement of excitable fields which allows unidirectional propagation of chemical pulses depending on the relative position of the excitable fields and the size of the gap of the diffusion field between them. The unidirectional propagation obtained by Motoike et al. [24,25] and Agladze et al. [27] was obtained by exploiting the geometry of active excitable fields in the presence of a gap between them.

Detailed analysis has been made of the mechanism leading to unidirectional propagation. We have presented quantitative results for one particular experimental arrangement and have shown how its behavior depends on both the excitability in the medium and its size. We have demonstrated the importance of the wave front spreading outside the excitable track and its orientation when it impinges on a constriction on its path. The interplay between these factors determines the accumulated activity at the point past the constriction and gives rise to propagation when the excitation exceeds the threshold.

Financial support from DGES Projects No. PB97-0540 and No. BXX2000-0638-C02-01 and MCyT Project No. BFM2000-0348 is greatly acknowledged.
[1] Waves and Patterns in Chemical and Biological Media, edited by H.L. Swinney and V.I. Krinsky, special issue of Physica D 49, 1 (1991).

[2] R.A. Gray and J. Jalife, Int. J. Bifurcation Chaos Appl. Sci. Eng. 6, 415 (1996).

[3] M.S. Spach, W.T. Miller, D.B. Geselowitz, R.C. Barr, J.M. Kootsey, and E.A. Johnson, Circ. Res. 48, 39 (1981).

[4] M. Gómez-Gesteira, G. Fernández-García, A.P. Munuzuri, V. Pérez-Muñuzuri, V.I. Krinsky, C.F. Starmer, and V. PérezVillar, Physica D 76, 359 (1994).

[5] J. Starobin, Y.I. Zilberter, and C.F. Starmer, Physica D 70, 321 (1994).

[6] K. Agladze, J.P. Keener, S.C. Müller, and A. Panfilov, Science 264, 1746 (1994).

[7] O. Steinbock, P. Kettunen, and K. Showalter, Science 269, 1857 (1995).

[8] M. Gómez-Gesteira, J.L. del Castillo, M.E. Vázquez-Iglesias, V. Pérez-Muñuzuri, and V. Pérez-Villar, Phys. Rev. E 50, 4646 (1994).

[9] J.M. Starobin and C.F. Starmer, Phys. Rev. E 54, 430 (1996).

[10] M.S. Spach, W.T. Miller, P.C. Dolber, J.M. Kootsey, J.R. Sommer, and C.E. Mosher, Circ. Res. 50, 175 (1982).

[11] A. Kadish, M. Shinnar, E.N. Moore, J.H. Levine, C.W. Balke, and J.F. Spear, Circulation 78, 1478 (1988).

[12] J. Brugada, L. Boersma, Ch. Kirchhof, and M. Allessie, Rev. Esp. Cardiol. 43, 558 (1990).
[13] Z. Noszticzius, W. Horsthemke, W.D. McCormick, H.L. Swinney, and W.Y. Tam, Nature (London) 329, 619 (1987).

[14] E. Dulos, J. Boissonade, and P. DeKepper, Physica A 188, 120 (1992).

[15] O. Steinbock and P. Kettunen, Chem. Phys. Lett. 251, 305 (1996).

[16] V. Gáspár, Gy. Bazsa, and M.T. Beck, Z. Phys. Chem. (Leipzig) 264, 43 (1983).

[17] I. Sendiña-Nadal et al., Phys. Rev. E 58, R1183 (1998).

[18] T. Yamaguchi, L. Kuhnert, Zs. Nagy-Ungvaray, S.C. Müller, and B. Hess, J. Phys. Chem. 95, 5831 (1991).

[19] R.J. Field and R.M. Noyes, J. Chem. Phys. 60, 1877 (1973).

[20] J.J. Tyson and P.C. Fife, J. Chem. Phys. 73, 2224 (1980).

[21] H.-J. Krug, L. Pohlmann, and L. Kuhnert, J. Phys. Chem. 94, 4862 (1990).

[22] A.R. Mitchell and D.F. Griffiths, The Finite Difference Method in Partial Differential Equations (John Wiley \& Sons, Chichester, England, 1997).

[23] M. deCastro, M. Gómez-Gesteira, and V. Pérez-Villar, Phys. Rev. E 57, 949 (1998).

[24] I. Motoike and K. Yoshikawa, Phys. Rev. E 59, 5354 (1999).

[25] I.N. Motoike, K. Yoshikawa, Y. Iguchi, and S. Nakata, Phys. Rev. E 63, 036220 (2001).

[26] A. Tóth and K. Showalter, J. Chem. Phys. 103, 2058 (1995).

[27] K. Agladze, R.R. Aliev, and T. Yamaguchi, J. Phys. Chem. 100, 13895 (1996). 